\title{
VR FOR ASSEMBLY TASKS IN THE MANUFACTURING INDUSTRY- INTERACTION AND BEHAVIOUR
}

\author{
Y. Eriksson ${ }^{1, \otimes}$, M. Sjölinder ${ }^{2}$, A. Wallberg ${ }^{2}$ and J. Söderberg ${ }^{2}$ \\ ${ }^{1}$ Mälardalen University, Sweden, ${ }^{2}$ RISE, Sweden \\ $\square$ yvonne.eriksson@mdh.se
}

\begin{abstract}
A testbed was developed aiming to contribute to further knowledge on what is required from a VR application in order to be useful for planning of assembly tasks. In a pilot study the testbed was tested on students. The focus of the study was to explore the users' behaviour, and to gain a better understanding of their experience using VR. The students experienced a gap between the real world and VR, which confirms theories that VR is not a copy or twin of an object or environment.
\end{abstract}

Keywords: human behaviour, virtual reality (VR), case study

\section{Introduction}

Technologies that has attracted a lot of attention over the last decade is virtual reality (VR) and Augmented Reality (AR). However, this study is limited to an interactive VR testbed. VR offers the possibility to create objects and environments that give the illusion of being real, making it possible to interact with objects, move about, and explore environments with a strong sense of presence. However, VR does not offer any haptic feedback besides vibration, if not additional devices are used. This represents a limitation when it comes to using VR for purposes such as interactive training and/or learning for assembly tasks, since assembly is a physical performance which necessitate ergonomic considerations such as weight and complexity in the actual task.

The aim of the pilot study presented in this paper is to contribute to further knowledge on what is required from a VR application in order to be useful for the learning and planning of assembly tasks in manufactory industry. Taking a multi-sensory approach to the development of a VR platform without using devices that give haptic feedback. The pilot study explored whether it was possible to simulate human perceptional cues within a training setting based on a VR environment.

We will discuss the possibilities to enhance and nuance feedback in the virtual experience through the use of sound, different visual cues, and limited haptic feedback which can be provided via hand control. This paper also discusses the consequences of VR as an environment, based on its own physical presuppositions in relation to the real environment.

\section{Previous research}

Great benefits can be gained if otherwise costly or dangerous work tasks can be practiced successfully in a VR environment. For instance, VR environments have been utilized for assembly task training (Brough et al., 2007). The user receives guidance on how to conduct different assembly steps, and 
feedback is given when errors occur. VR environments have also been developed with the aim of improving the design of assembly processes, based on existing design methods and the analysis of performance data (Sung et al., 2009). Several studies on VR for learning and training has been focused on simulation in VR, where natural human motions have been integrated into computer aided assembly planning (Seth et al., 2011). VR is highly relevant to the manufacturing industry as it offers the possibility to learn a process. A process learned in a virtual environment transfers well to performance in real life, as confirmed by study results showing a similar level of performance being achieved in conventional training compared to training in virtual reality. To be able to achieve high quality haptic feedback, expensive devices need to be used (Lim et al., 2007).

Using a testbed, it is possible to facilitate and enhance the implementation of VR in the manufacturing context. From there, we can gather knowledge on how and where in the supply chain this technology should be implemented in order to achieve the greatest productivity gains and/or cost efficiency.

However, given how quickly VR technology is evolving, along with new areas of usage, there is a need to develop a framework for interaction based on human perception, but within a context based on the rules within the VR environment (Rubio-Tamayo et al., 2017). Mental models used during interaction with our real environment (Johnson-Laird, 1983; Staggers and Norcio, 1993) might not be applicable, and there is a need to better understand when and why they fail. Scholars have defined what is required of a visual representation in order to be accepted as a representation of an object or milieu (Tversky, 2013; Bae and Watson, 2013; Eppler, 2013). Interplay between different perceptual cues are also well known and, in 1920, David Katz conducted a study where he compared touch with vision and came to the conclusion that, "When we touch some common object, the tactile impression is always permeated with visual experience" (Katz, 1989, p.156). It is necessary to consider that a visualization is a re- presentation, where something is presented with a specific intention that might not necessarily be understood by a user in the way intended (Rose, 2016). In addition, it is also a question of agreement between the visualization and the user, which can be described as "the beholders share" (Gombrich, 1982). This means that users need to contribute with their imagination and must accept the conditions for interaction with a given visual. However, there is a lack of research when it comes to understanding how mental conventionalization influences users' understanding and use of VR environments in relation to the real environment. Additionally, when conducting tasks in a VR environment, the interplay between different perceptual cues needs to be understood based on the specific roles that apply in the VR environment, and interaction with objects needs to be adapted to the presuppositions in this environment.

From a biological perspective, visuals constitute the largest proportion of all information we collect in order to gain situational awareness. An estimated $40 \%$ of the brain's cortex is used to process visual information, while only about $3 \%$ contributes to audio processing. Nevertheless, sound is extremely important, and can often mean the difference between an operational and dysfunctional VR application. Another difference between vision and sound is that we are more likely to benefit from audio information at a lower level of consciousness, while we become aware of visual events in a more obvious way. Sound puts us on standby, preparing us to act in new situations - before we even know it.

The representations in the VR testbed are to be regarded as engineering models. A VR model, like an engineering drawing, constitutes a language in itself, containing conventions and symbols that represent very specific details and expected actions. Just like in the interpretation of engineering drawings, experience is required. A VR interpreter needs to know how to use the instructions. Crucial to visual perception is the ability to detect relationships, as well as search for recognizable patterns (Arnheim, 1969). Visualizations can facilitate the interpretation or reading of a simulation, which can be defined as a set of techniques - not technology per se-for augmenting or replacing the physical experience. Even though VR is considered to be realistic, it has its limitations and the representations do not totally replace reality. How a simulation is experienced is dependent on the design of the visualization itself, as well as on the interaction design of the interface (Ware, 2012).

It is also dependent on the usage. VR is an altogether visual and aural medium, where it is possible to act and interact with the environment and its objects in ways that other mediums cannot provide. It is possible to lift, move, push, rotate, and throw imaginary objects, but the only confirmation the user receives that the interaction is taking place is what they can see and hear. 


\section{Testbed for assembly and planning}

In order to explore how VR can be used as a learning tool for assembly and planning of a production line, a testbed was developed. The testbed environment consists of two tables where assembly pieces and tools are laid out (see Figure 2). For the pilot study described in this paper, the assembly pieces constitute a simplified and scaled-down version of the cab of an Excavator made by Volvo Construction Equipment (see Figure 1).

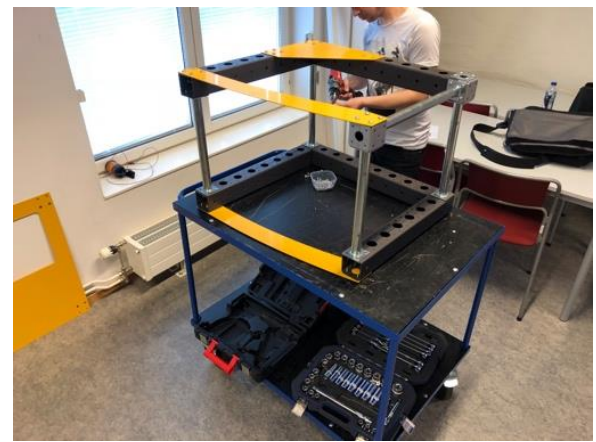

Figure 1. Scaled-down version of the cab of an excavator

The choose of the cab model as a base for the VR testbed was motivated since the university own the cab models and they do exist in three different varieties and are used by students in one of the civil engineering programs for training and planning of a single and a mixed assembly production line. The students practice assembly of the three different varieties of the cab and found out the most efficient way to assembly and from that, they plan the Takt time for every single sequence. The cab model is divided into 13 pieces, it takes 76 screws to completely assemble the model and, when fully assembled, it measures $63 \times 49 \times 70(\mathrm{LxWxH})$ centimetres. The basis for the $3 \mathrm{D}$ models of the pieces is an existing physical model of the same cab that the engineering students also worked with. Some custom-made parts had CAD-files that could be directly converted to be used in the testbed, while other pieces had to be modelled based on measurements of the physical pieces.

Apart from the pieces, there were two tools included in the pilot study environment. One tool was a tabletlike digital display with three buttons where the user could read instructions (e.g., how to retrieve screws and what the final product is supposed to look like). The other tool was a screwdriver used for attaching pieces (see Figure 2). When operated, the screwdriver provides aural and haptic feedback. It uses a sampled sound from a real drill, where the pitch and rotation speed is controlled by how hard the user presses the trigger button on the hand controller. Like many real screwdrivers, it also has a slip clutch mechanism that stops the rotation and starts a stronger haptic rumble effect in the hand controller when a screw has been tightened completely. Technically, the testbed was created using the Unreal Engine, developed by Epic Games. It supports development for many platforms, including systems from major VR hardware vendors like Oculus and HTC. For the pilot study, we had two different setups: one using Oculus Rift with Touch controllers, and the other using HTC Vive Pro with Vive controllers. Both types of controllers worked in a similar way, where the side grip button was used to grab objects and tools while the trigger button, operated with the index finger, was used to control the speed of the screwdriver.
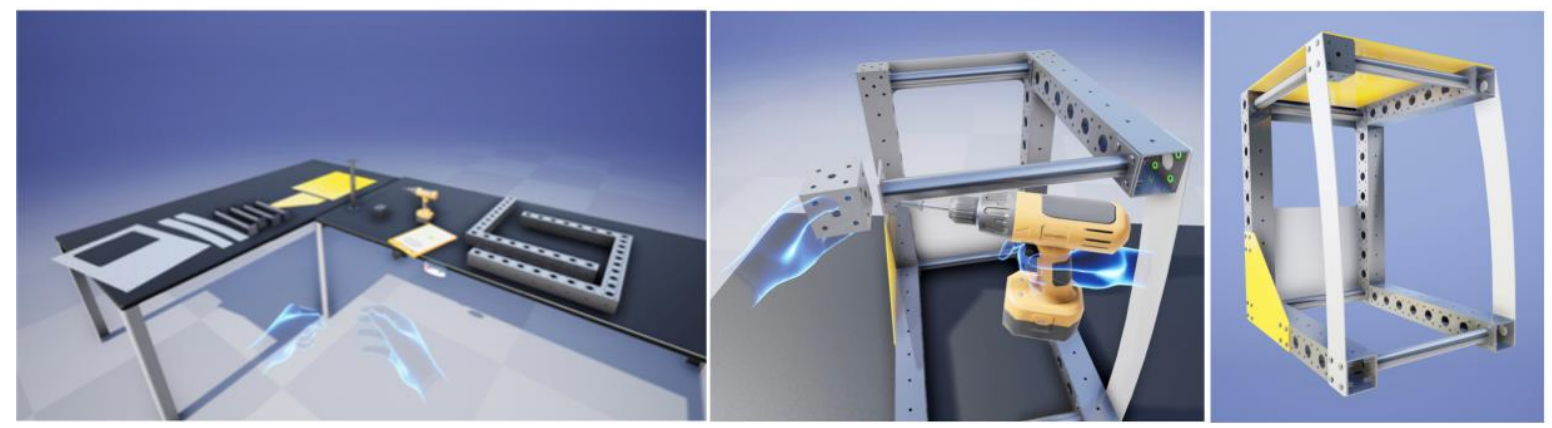

Figure 2. The VR-model 


\section{Pilot study}

As part of the engineering program at the university where this pilot study took place, students learn about assembly conditions and how to plan a single and mixed product line (two variations of a single product) by using a manufactory lab consisting of three different simplified and scaled-down models of construction equipment cabs. The students are expected to figure out the best way to assemble the cabs and determine the Takt time for assembly for both the single and flexible product lines. The cab models allow students to practice assembly and measure the time needed to complete each step of the assembly sequence. The task they were given was to design and build an assembly line for one of the cab types (single-product assembly line). In order to calculate the Takt time, the students needed to execute each assembly moment several times. The manufactory lab contains three versions of the cab models, with one cab of each model. Since the typical student groups are around twenty students divided into sub-groups of three to four members each, the cab models that have been used for almost ten years include parts such as screws and threads that have become worn out. In a pilot study the VR-testbed, developed based on the training environment described above, was tested on engineering students. The focus of the study was to explore the users' behaviour, and to gain a better understanding of their experience using VR for training.

\subsection{Participants}

As the first step of evaluating the testbed, a pilot study was conducted on 21 fourth year engineering students. The age of the students was between 21 and 36 years. Six of the students were female and 15 were male. A total of 14 of the 21 students had limited or no experience of VR, with some having experienced VR only once before. Several of the students were participating in a parallel course with a focus on visualization, in which they had the opportunity to test VR for less than 10 minutes each. Six of the seven students with former VR experience had played games, and one student had experienced VR in a working context. Six of the students had previous work experience as an assembler in an assembly line (see Table 1).

Table 1. Pilot study participants' age and previous experience with VR and assembly tasks

\begin{tabular}{|l|l|l|l|}
\hline & Male & Female & All \\
\hline Number of participants & 15 & 6 & 21 \\
\hline Age & 24.33 & 27.17 & 25.14 \\
\hline VR experience & & & \\
\hline None or one time & 10 & 4 & 14 \\
\hline Games & 4 & 2 & 6 \\
\hline Working context & 1 & & 1 \\
\hline Experience working in an assembly line & & & \\
\hline None/school & 10 & 5 & 15 \\
\hline Working context/internship & 5 & 1 & 6 \\
\hline
\end{tabular}

\subsection{Method}

Ethnographic methods were used for this study, including observations of participants' performance followed by individual and group interviews. Every single student received a brief introduction to the equipment, after which they used the platform for 15 minutes. During this part of the session, they assembled as much of the model as they could within the time frame. No further instructions were given since one important aspect was to see how they approached the assembly task in the VR-environment. This was followed by a 10 minutes interview where each student was asked a total of seven questions. Approximately half of the students did not speak Swedish, so in those cases the interview was conducted 
in English. The test took place over the course of two days, and two parallel setups were used with one instructor at each platform. Interviews were conducted in parallel by two different researchers.

During interviews, the first question that was asked was if the student had had any previous experience with VR or had previously worked as an operator or assembler. This was followed by questions related to the course they were taking, which was focused on planning for single and flexible product lines. These questions included how far the student had come in the group with the assembly planning task, their opinion about learning to plan in VR, in what way the VR milieu was an advantage or disadvantage in relation to the real cab/object/environment, what parts in an assembly task should be supported or not through the use of VR, and whether there were parts of the task where only using VR could be enough. The students were also asked if they followed the same procedure for different moments, just as they normally do when assembling a real cab.

The empirical data for this study, related to the questions, was collected individually by the two interviewers. We have grouped the individual answers and opinions in relation to the questions in order to gain an overview of the students' collective experience using VR. The qualitative analysis was conducted by identifying themes and map the answers to a relevant theme.

\subsection{Results}

During the interviews, participants were asked what they thought about learning how to plan an assembly line with the aid of VR. Furthermore, they were asked about what they thought were the advantages and disadvantages of VR compared to learning in a real environment, as well as where in the process they thought VR could be useful compared to using real models. A qualitative analysis was conducted, and themes were identified based on participant answers. The results are described according to the following themes: 1) Environment and interaction, 2) Differences between using VR and a real model, 3) Using VR for learning and planning assembly tasks in a university course and 4) Phases and tasks where VR could be useful in a manufacturing context. The comments made by several students have been considered as main results (see Figure 3).

The main results with respect to environment and interaction were:

- Need for getting familiar with the VR-environment before it can be used for learning assembly tasks

- Lack of weight and gravity challenged existing perception of interaction with objects

- Objects moved and it was hard to get a grip of them

The main results with respect to differences between using VR and a real model were:

- No consensus about similarity with real world experiences

- Assembling tasks are faster and it is easier to correct mistakes in a VR-environment

- No risk of destroying things in a VR-environment

- Easier to get objects in place and no need for someone else to hold parts or assist

- Due to lack of weight - difficult to understand real usage context

- Not possible to estimate real takt times since assembly is faster in a VR-environment

The main results with respect to using VR for learning and planning assembly tasks in a university course were:

- VR is better than theoretical learning and it can be used before real world learning

- VR could support the ability to plan and provide the possibility to learn order of different steps

- A physical provide better insights about things that can go wrong

- VR cannot be used for learning to estimate takt times

The main results with respect to phases and tasks where VR could be useful in a manufacturing context were:

- The students thought it would be good to have a combination of real life and VR training

- Useful in the beginning of the learning and for getting familiar with a task

- VR could be used when planning the production line for a new product

- VR could be suitable for the early stages of prototyping since it provides a good overview

Figure 3. List of topics based on comments from several of the participants 


\subsubsection{Environment and interaction}

The students found the VR application and graphics to be smooth, and thought it was fun-like playing a game. However, the students expressed that they needed more time to practice in the VR environment and that, before using VR for planning, developing experience on how to use VR would be necessary. It was pointed out explicitly, by eight students, that it takes some time to get used to interacting with VR equipment and become familiar with it. One of the students said: "It is hard to learn VR as such, the lack of VR experience made it hard to assemble." Another student said, "It was interesting, but you need someone to help you understand how the VR environment works." The students also pointed out that the VR environment needs to be used a couple of times before you can get into learning the assembly tasks. Once they had grown more familiar, they thought it was easier to remember the assembly instructions.

With respect to interaction, the students pointed out issues related to the lack of weight attributed to objects, as well as to items moving while they were trying to tighten the screws due to a misperception of weight. This was pointed out explicitly by three students. The observation that everything was very "movable" and easy to push unintentionally was explicitly mentioned by two students. However, the issues related to lack of weight and the objects moving was mentioned by several students over the course of the entire interview. The lack of weight was most pronounced with respect to heavy parts, where the difference compared to real life was greatly apparent. Furthermore, the students mentioned that objects (the screwdriver and tablet with instructions) moved and fell to the floor as they were trying to place them into a position due to the lack of gravity compared to real life where everything is stable. Furthermore, they could not get a grip on things - especially thin objects. Some objects were thin, and others were not, and it was difficult to learn how to lift them. The students also said that it was difficult to assess the size of objects and estimate distance differences.

In general, the students thought that using VR was a nice experience. Particularly in regard to having the opportunity to try new things and the ability to be more careless since it was okay to drop tools and other things on the floor without breaking them. For example, one student said, "It was good that it was possible to make mistakes without consequences."

\subsubsection{Differences between using VR and a real model}

Several students felt that the VR testbed was quite close to reality, while others declared that they found it interesting but that it was far from real life. Some of the students said that the VR environment did not feel like a real environment and they experienced it very differently - this was explicitly pointed out by five students. Some of the students also found the VR environment unrealistic due to the lack of similarities with real world interaction, and therefore felt isolated in the VR world.

Some important aspects were discussed by the students regarding the advantages of using VR. The students said that it did not take much time to complete the assembly task since they did not have to deal with details that require a lot of energy, and they did not have to carry heavy parts. In this study, six of the students said that VR learning was not as time consuming as learning in a real environment, especially when something goes wrong. Furthermore, they mentioned that it was possible to carry out the tasks without previous experience since there was no risk of destroying anything. They also emphasized that it was easier to get objects in place in the VR model and that there was no need for someone else to assist with holding them during assembly. Finally, the students found it easier to determine where to place different parts, and to test different things in the VR environment.

With respect to the disadvantages, some of the students thought that it was difficult to understand the real usage context and object weight (mentioned by seven students). Also, the screws would hit the correct position immediately and get into place too quickly which, in turn, make it difficult to estimate the real Takt times (mentioned by five students). In general, the lack of time experience was mentioned by several of the students throughout the interview. When working on a physical model, you experience the amount of time you have spent. In the VR testbed, the process of screwing a part into place, for example, goes much faster. One of the students mentioned that "everything is faster, but you miss the physical feeling and it is harder to measure." Another student phrased it this way: "You do not get the Takt time. Weight, tools for fixation, and security thinking is missing." Additionally, it was mentioned by the students that they found it impossible to estimate time for sequences requiring 
two operators. Finally, a further disadvantage that was mentioned was a lack of ergonomics in relation to the absence of weight and poor physical perception.

\subsubsection{Using VR for learning and planning assembly tasks in a university course}

Even though the students lacked VR experience, they found value in learning the various assembly moments in VR. Two of the students explicitly said that VR is better than theoretical learning and that it is good to conduct assembly tasks in VR prior to real world learning. It could increase the ability to plan and serve as a good way to practice and learn the order of different steps before testing it out in real life. Since the physical cab models were partly worn out, with bad screws and a broken screwdriver, the students thought that it would be easier to use a VR model for learning. The use of a VR model also reduces the risk of getting injured, and makes it is easier to get a feeling for the environment around the assembly line. The students also had some speculation regarding the learning process, where they thought it was probably easier to learn how to do something in VR than in real life. "It is easier to remember while assembling in VR," said one student. "In VR you do not have to do shitty things like dealing with a bad screw, and you get a better overview."

However, the students thought that the use of a physical model provides a better understanding of what could go wrong in relation to, for example, the process of screwing a part into place. Additionally, in the VR testbed it was not possible for the students to perceive and understand the lack of space when assembling details in the same way that they do with the dummy product cab they use in the course. Again, the students pointed out issues related to time planning, and that it must be difficult to learn how to plan Takt time for each step of an assembly task in VR.

In general, the students stated that, with better training, VR has many advantages and that they wanted to learn more about and how to use VR for their future work life. Given the potential advantages of VR for the future, the students hope that training with VR will become more accessible. Especially since it is possible to save a lot of time by training with VR, as you do not need to disassemble in order to reassemble every time you practice an assembly task.

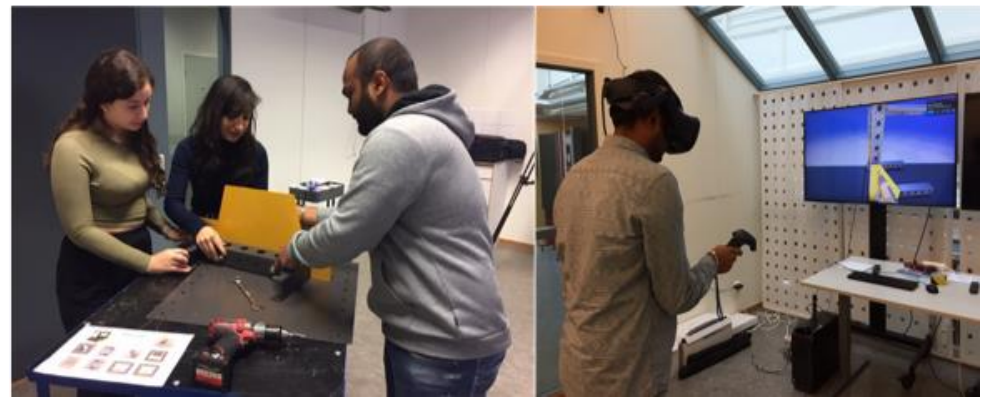

Figure 4. Students conducting the test

\subsubsection{Phases and tasks where VR could be useful in a manufacturing context}

In general, the students thought it would be good to have a combination of real life and VR training when learning or planning new assembly tasks. They thought that it could be supportive to start training assembly tasks in VR before conducting them in real life (explicitly mentioned by three of the students). They also brought up the subject of safety (explicitly mentioned by two of the students), and that it would be helpful if, in order to convey risks, there were consequences in VR just as there are in real life. There was agreement among the students regarding the usefulness of VR. They thought it would be great for training and the planning of assembly tasks (explicitly pointed out by four students). They recognized VR as a helpful environment for becoming familiar with a task. The students also thought VR will be useful for finding out what order of steps in a work task sequence will work best (explicitly pointed out by four students). For a new product, VR would support the ability to understand what to do and what task sequences to conduct. Several of the students brought up the advantage of using VR for creating something new and that it is a good tool for testing different parts. Seven of the students pointed out that VR would be useful for gaining an overview of a process and provide support when it comes to planning the different steps. One student mentioned that he had 
quoted something he'd heard in a lecture when he said VR would be helpful for "learning the order of how to do things." He also said that he "would like to see the whole factory environment in VR, with trucks you should pay attention to and tools that should be used."

Most of the students thought that VR would be suitable for the early stages of prototyping, since it provides a good overview, before moving on to a physical model. Others thought that VR would be suitable and useful during later stages and steps when testing how a product would work tangibly and physically. Some of the students thought VR would be most suitable for simulating an assembly task involving larger parts. Furthermore, the students mentioned that VR could help increase the understanding of an assembly task in the case where there is no access to physical objects, and that it could be used for learning how to put complex parts together. VR could also reduce the burden on the "real" model in terms of also providing a number of VR software models. Finally, it was suggested that VR could be a nice tool for selling products and demonstrating how easy it is to assemble them.

The students thought VR would be less useful when measuring accurate timing, and when trying to understand which moment in an assembly task needs to be fixed. One student stated, "The gravity is missing, that was disturbing. I missed having a guide that showed the various steps. There is a problem with the screws in reality, but not in VR." Finally, some students said that VR would not be useful in situations that entail safety risks, since it is hard to understand these risks in a virtual environment.

\section{Discussion}

It does exist a lot of assumption regarding the digital native generation, in relation to that it is surprising that the student had very limited experience of VR. In education setting it is necessary to be aware of what steps are required in order to introduce VR environment. Firstly, the student needs to be introduced to the tool as such, and secondly how to navigate in a virtual world and especially how to perform. One important insight from the pilot study is that the students missed the sensation of weight in VR, which is due to the absence of gravity. The parts that were to be assembled were weightless and easy to turn around, which provided zero understanding of their real weight. On the other hand, the screwdriver was weighted and fell to the floor when participants let go of the grip button. It is worth considering that the participants possessed previous experience assembling the real cab model, and therefore might have known how it should feel to assemble the different parts. To some extent, the problems the students encountered could be explained by their mental image or model of the tasks they expected to perform and the conditions they expected to perform them in with regards to what was possible in the VR environment (Johnson-Laird, 1983; Staggers and Norcio, 1993). Since VR is not a mirror of the real environment, perceptual and cognitive frameworks adapted to this environment are needed for successful interaction (Rubio-Tamayo et al., 2017). This indicate that the situational awareness based on visual input were not matched by the mental image of the physical cab. In this case, the conflict with physical rules from the real world might have been even stronger due to the fact that the VR environment itself was inconsistent in terms of some of the objects being weighted and others not. The fact that the students dropped the screwdriver could be further explained by their interaction with the tool. The only way an individual could control the screwdriver was by pressing the trigger button of the controller with their index finger. When letting go of the trigger button, the experience was that they were still holding the screwdriver when, in fact, they were holding the controller. This interaction might have caused a clash between the participants' visual perception and experience and their tactile experience (Jackson et al., 2009). However, a sound was connected to the screwdriver in order to give feedback to the user. No one mentioned the sound. It seems that it was embedded in the understanding of the use of the tool in VR. The students perceived a mismatch in relation to size between different parts of the cab in VR, and they also found it difficult to estimate distance. This could be explained by their lack of experience with how things were represented in VR and the fact that they did not know what pattern to look for (Arnheim, 1969). Additionally, the VR representations did not resemble traditional engineering drawings (Feurgeson, 1994).

The instructions provided on how to use the VR platform were limited, and that is because we wanted to explore to what extent the students would be able to complete an assembly task in VR without any further instructions. This resulted in too large a burden on the students, they needed to understand how to use VR and how to assemble the cab. Interestingly, they did not use the same strategy as they would 
with the real cab, which might support the conclusion that there was a gap between interaction in the VR environment and in the real world. However, the students agreed on the advantages of using VR for learning and planning assembly, though it should be noted that this was something that they had learned in a parallel course they were taking.

With respect to interaction design, the sensation of weight in a VR environment is a challenge and several design decisions need to be made. The sensation of weight can be achieved through the use of active haptic devices attached to the ground. Through these, the system may apply forces directly onto the user's body. These typically have limited working room, making them unsuitable for larger movements in a VR environment. Ungrounded dynamic feedback, in the form that may be integrated in a handheld controller, can provide some feeling of resistance, inertia, and shiftable weight [Zenner and Krüger, 2019]. However, ungrounded devices lack the ability to convincingly render the sensation of a grasped object's weight. Both ungrounded and, especially, grounded haptic devices add complexity to the setup and are neither as inexpensive, robust or readily available at scale as the current crop of VR controllers. Dropping the ability to experience weight and using only standard controllers, research has been conducted on instead conveying object weight visually through how they behave while moved (e.g. Weser and Proffitt, 2019). In these tests, a limit is placed on how fast objects can be moved or dragged based on how heavy they are. Heavier objects move more slowly than lighter ones, trailing the virtual hand when moved quickly. Moving a heavy object too fast may also cause it to be dropped. By representing weight visually and providing virtual reference objects with known weights, a user may learn to identify object weights based on their behaviour. However, in the pilot study where the task entailed carefully aligning pieces to be screwed together, using this technique of having lag time between controller movement or action and what objects do in response was deemed undesirable.

Regarding the difficulties the students encountered when objects moved unintentionally-normally, when tools collide with pieces during the assembly of physical models, the operator immediately picks up on that fact due to haptic feedback, and either stops the movement or pushes through depending on the desired outcome. In the testbed, collisions trigger a haptic rumble in the controllers but a novice user or a user focusing elsewhere may miss this and inadvertently bump and move pieces that had been carefully positioned. Several students in the pilot study struggled with this in the beginning of the sessions. However, over time, this became less and less of a problem as they gained more experience interacting in VR and took time to be more careful during those operations. In the current version of the testbed, the position of the hands and, by extension, the objects held in the hands, exactly match the movement of the controllers. This was done to enhance the feeling of direct manipulation and to support fine and rapid movement of the pieces during assembly. A more forgiving alternative could be to design the system so that a held object that collides with other stops at the point of collision and then move slightly out of grasp. This could potentially help during delicate operations at the cost of the user feeling less in control of the objects, since held objects may visually move away from the hand holding them.

\section{Conclusion}

The testbed, based on the cab of an Excavator made by Volvo Construction Equipment, was designed in order to explore the potential of using VR for the learning and planning of assembly tasks. Since the dummy of the cab is currently used in the engineering education program, conducting the pilot study with students as participants is motivated. All of the students had prior experience with the assembly and planning of both single and flexible product lines. When the students used the VR application, they already had experience of working with the physical cab. In the pilot study, it was obvious that the students experienced a gap between the real world and VR. This contradicts the common idea that VR duplicates the physical environment or objects in it and is often regarded as some kind of twin to a specific object or environment. Instead, the study confirms theories that VR is a re-presentation of an object and/or environment, meaning that it visualizes aspects such as appearance, relationships and size, much like 2D visualizations do. A VR environment exists with its own set of conditions, but it is possible to discover a relationship to the physical world.

The students participating in the pilot study gained practical VR experience beyond a theoretical standpoint. Participating in the study provided them with a new perspective. However, the pilot study 
also raised new questions regarding what is required in order to move between the real world and the virtual one. What mental schemata are needed? And how can VR be used for product planning? The pilot study indicates that there is much more to be learned about how VR can be used in manufactory industrial settings.

\section{References}

Arnheim, R. (1969), Visual Thinking, University of California Press.

Bae, J. and Watson, B. (2013), "Toward a Better Understanding and Application of the Principles of Visual Communication", In: Huang, W. (Ed.), Handbook of Human Centric Visualization, Springer Verlag.

Brough, J.E. et al. (2007), "Towards the development of a virtual environment- based training system for mechanical assembly operations", In: Proceedings of the 1st international virtual manufacturing workshop.

Eppler, M.J. (2013), "Knowledge visualization", In: Marchese, F.T. and Bannisi, E. (Eds.), Knowledge Visualization Currents, Springer Verlag.

Feurgeson, E. (1994), Engineering and the Mind's Eye, Mass, Cambridge.

Gombrich, E.H. (1982), The Image and the Eye: Further Studies in the Psychology of Pictorial Representation, Phaidon, Oxford.

Jackson, S.R. et al. (2009), "There may be more to reaching than meets the eye:re-thinking opticataxia", Neuropsychologia, Vol. 47 No. 6, pp. 1397-1408.

Johnson-Laird, P.N. (1983), Mental Models: Towards a Cognitive Science of Language, Inference, and Consciousness, Cambridge University Press, Cambridge.

Rubio-Tamayo, J.L. and Gertrudix Barrio Francisco García García, M. (2017), "Immersive Environments and Virtual Reality: Systematic Review and Advances in Communication", Interaction and Simulation Multimodal Technologies Interact, Vol. 1 No. 4, pp. 21. https://doi.org/10.3390/mti1040021

Katz, D. (1925/1989), The World of Touch, Questia, com.

Lim, T. et al. (2007), "Factors affecting user performance in haptic assembly", Virtual reality, Vol. 11 No. 4, pp. 241-252. https://doi.org/10.1145/3290605.3300441

Rose, G. (2016), Visual Methodologies. An Introduction to Research within Visual Materials, Sage Publication Ltd, London.

Seth, A., Vance, J.M. and Oliver, J.H. (2011), "Virtual reality for assembly methods prototyping: a review", Virtual reality, Vol. 15 No. 1, pp. 5-20.

Staggers, N. and Norcio, A.F. (1993), "Mental models: concepts for human-computer interaction research (PDF)", International Journal of Man-Machine Studies, Vol. 38 No. 4, pp. 587-605.

Sung, R.C.W. et al. (2009), "Automated design process modeling and analysis using immersive virtual reality", Computer-Aided Design, Vol. 41, pp. 1082-1094.

Tversky, B. (2013), "Visual thought", In: Huang, W. (Ed.), Handbook of Human Centric Visualization, Springer-Verlag, New York.

Ware, C. (2012), Information Visualization: Perception for Design, Morgan Kaufman.

Weser, V. and Proffitt, D.R. (2019), "Making the Visual Tangible: Substituting Lifting Speed Limits for Object Weight in VR", PRESENCE: Virtual and Augmented Reality, Vol. 27, pp. 68-79.

Zenner, A. and Krüger, A. (2019), "Drag:on - A Virtual Reality Controller Providing Haptic Feedback Based on Drag and Weight Shift", In: CHI Conference on Human Factors in Computing Systems Proceedings (CHI2019), May 4-9, Glasgow, Scotland UK. ACM, New York, NY, USA, p. 12. 